\title{
The Effect of Bispectral Index Monitoring during Induction of Anesthesia on the Amount of Propofol Consumtion in Patients Candidates for Surgery
}

\author{
Borjian Boroojeny Shahram", Seyedeh Maryam Hojjat ${ }^{2}$
}

\author{
${ }^{1}$ Assistant of Professor, Department of Anesthesia, Zahedan University of Medical Sciences, Zahedan, Iran. \\ 2Resident of Anesthesology, Medical School, Zahedan University of Medical Sciences, Zahedan, Iran. \\ *Correspondence to: Borjian Boroojeny Shahram (E-mail: borjianboroojenyshahram@yahoo.com) \\ (Submitted: 06 April 2021 - Revised version received: 21 April 2021 - Accepted: 13 May 2021 - Published online: 26 August 2021)
}

\begin{abstract}
Introduction Propofol is a new intravenous hypnotic drug that is widely used in anesthesia and monitoring the depth of anesthesia. BIS is a parameter derived from electroencephalography that is associated with sleep deprivation and loss of consciousness. This study investigated the effect of Bispectral index (BIS) during induction of anesthesia on the amount of propofol consumed in patient candidates for surgery.

Materials and Methods This double-blind clinical trial study was performed on patients undergoing elective surgery under general anesthesia in a hospital. Patients were divided into case and control groups. After being transferred to the operating room, patients were monitored including ECG 3 or 5 leads, non-invasive barometer and pulse oximetry. Mean arterial blood pressure and heart rate were measured before and after induction, immediately and 5 minutes after intubation. The dose of propofol was then measured. Data analysis was performed by SPSS software version 20.

Results In the present study, no significant difference was found between the mean age and gender $(P>0.05)$. The amount of propofol consumed in the case group was significantly lower than the control group $(P=0.039)$ and the amount of propofol consumed in men and women was not statistically significant $(P<0.05)$. Mean arterial blood pressure before induction was not statistically significant between the two groups $(P=0.83)$. However, a statistically significant difference was found in the mean arterial blood pressure of the patients during the 4 time points $(P=0.001)$. There was no statistically significant difference in heart rate between patients before induction $(P=0.48)$. Statistical analysis of data by ANOVA test did not show a significant interaction between time and group $(P=0.418$ and $P=0.74)$. However, a statistically significant difference was found in patients' heart rate during the 4 time points $(F=7.59$ and $P=0.001)$. Moreover, a significant increase was observed in heart rate after intubation in both groups $(P=0.001)$.

Conclusion The use of BIS can be effective in reducing the amount of propofol consumed and its side effects. The condition of patients under general anesthesia can be improved by BIS, resulting in improvement of their subsequent condition.

Keywords Bispectral index (BIS) monitoring, propofol, general anesthesia
\end{abstract}

\section{Introduction}

Maintenance of anesthesia during surgery can be done using inhaled or intravenous drugs. Intraoperative doses of these drugs are often increased or decreased based on hemodynamic symptoms, such as blood pressure and heart rate. This is due to the relatively direct relationship between the depth of anesthesia and the amount of blood pressure, so blood pressure can be used as a reliable monitoring of the depth of anesthesia. ${ }^{1}$ Maintaining blood pressure within the standard range is one of the most important tasks of an anesthesiologist during surgery. Bispectral Index (BIS) is a parameter derived from electroencephalography, which is a number between 0 and 100 based on the brain waves recorded from the frontal lobe of the brain and their frequency and the prevalence of EEG flat time. The best depth of anesthesia indicated by BIS when using propofol is between 40 and $60 .^{2-4}$ Propofol is the most common drug for inducing intravenous anesthesia and one of the most widely used drugs for maintaining anesthesia in the operating room. In animal and human studies, hypotension during anesthesia has several complications such as risk of heart attack, stroke, cognitive or memory impairment, kidney damage, etc., ${ }^{5-8}$ Propofol is classically administered on an $\mathrm{mg} / \mathrm{kg}$ basis and one of the most common side effects of propofol is hypotension. ${ }^{9}$ The use of BIS may prevent over-administration of propofol while achieving sufficient depth of anesthesia and thus prevent complications such as hypotension.

Propofol is a first-line drug in intravenous anesthesia. In complete intravenous anesthesia, a combination of propofol and a drug is used to induce and maintain anesthesia. ${ }^{10}$ It is a hypothetical drug with an unknown mechanism, but it is capable of activating chlorine channels and inhibiting synaptic inhibition by stimulating the beta component of a GABA system. Its ED50 is $1-1.5 \mathrm{mg} / \mathrm{kg}$ following a bolus injection. The amount of anesthesia is dose dependent and it is able to convert the alpha rhythm of awakening to the dominant beta rhythm in case of infusion with a dose of $100 \mathrm{mcg} / \mathrm{kg} / \mathrm{min} .^{11,12}$ The EEG spectral analysis shows an almost good correlation with movement during surgical stimulation. ${ }^{13,14}$ This method does not monitor the depth of anesthesia, but it can predict the patient's movement if the level of anesthesia is insufficient. BIS is the best predictor of motion relative to plasma propofol concentration or other EEG criteria such as spectral margin or median frequency. ${ }^{15}$ Therefore, according to the above, this study was conducted to investigate the role of BIS in reducing propofol consumption during induction of anesthesia while maintaining sufficient depth of anesthesia. 


\section{Materials and Methods}

This double-blind clinical trial was performed on patients who were candidates for elective surgery and underwent general anesthesia in hospitals affiliated to Zahedan-Iran University of Medical Sciences in 1397-98. Patients entered the study after obtaining informed consent and having inclusion and exclusion criteria. Inclusion criteria include: having informed consent, ASA Class I \& II, age between 18 and 65 years. Exclusion criteria include: intubation history, decreased level of consciousness for any reason before surgery, brain surgery, cardiovascular disease, esophageal reflux, hiatal hernia, liver or kidney failure, allergy to propofol, taking sedatives or drugs 24 hours before surgery, history of any neurological disorders and use of psychiatric drugs, patient history of addiction, anemia with hemoglobin less than $10 \mathrm{mg} / \mathrm{dl}$, patient dissatisfaction with participation in the study.

\section{Sample Size}

The sample size was calculated using the formula and, ${ }^{16}$ at least 40 people in each group.

$$
\begin{gathered}
n=\frac{\left(Z_{1-\alpha / 2}+Z_{1-\beta}\right)^{2}\left(S_{1}^{2}+S_{2}^{2}\right)}{\left(\mu_{1}-\mu_{2}\right)^{2}} \\
n=\frac{(1.96+1.64)^{2}\left(98.6_{1}^{2}+206.5_{2}^{2}\right)}{(397.8-534.4)^{2}}=36.4 \approx 40
\end{gathered}
$$

Propofol intake in BIS group: (397.8 \pm 98.6)

Propofol consumption in non-BIS group: $(534.4 \pm 206.5)$

\section{Procedure}

Patients were randomly selected and then divided into case groups (BIS) and control using random blocking method. After being transferred to the operating room, the patient underwent monitoring including ECG 3 or 5 leads, Noninvasive Blood Pressure (NIBP) and pulse oximetry. The initial information of the patient was recorded and then the venous cannula No. 20 was implanted in one of the patient's hands and Ringer serum was infused at $4 \mathrm{cc} / \mathrm{kg}$ within 10 to 15 minutes. In the study group, the leads of the BIS device (Iran Electronics Industries) were connected to the foreheads of the patients. Induction of anesthesia was performed with 1-2 mg/kg fentanyl and 1\% propofol (Fresenius-Kabi, Germany) at a dose of $2 \mathrm{mg} / \mathrm{kg}$ at a rate of $130 \mathrm{mg} / \mathrm{min}$. In the case group (BIS), after 20 seconds, propofol was injected at the rate of $0.2 \mathrm{mg} / \mathrm{kg}$ if the BIS does not reach less than $60 \%$, and this cycle continued until the BIS decreased to less than $60 \%$. In the control group, 20 seconds after propofol injection, in case of no apnea or loss of eyelash reflex, propofol was injected at the rate of $0.2 \mathrm{mg} / \mathrm{kg}$ and this cycle was continued until complete anesthesia, then Cisatracurium with dose $0.15 \mathrm{mg} / \mathrm{kg}$ was injected and endotracheal intubation was performed after 3 minutes. Maintenance of anesthesia was continued with propofol at a dose of 4-8 $\mathrm{mg} / \mathrm{kg} / \mathrm{h}$. Estimation of anesthesia in the patient in the clinical method included loss of oral response, loss of sense of gentle touch and loss of eyelash reflex.

The dose of propofol in each group was calculated. MAP blood pressure and heart rate of patients were measured before induction, after induction, immediately after intubation and
5 minutes after intubation. In case of any unusual event such as laryngeal spasm or arrhythmia that required treatment outside the above protocol, the patient was excluded from the study while recording the event.

\section{Data Analysis}

The information was displayed in the form of tables and graphs. Data analysis was performed according to the type of variable and the required conditions by appropriate statistical tests (including Chi-square, Mixed ANOVA, Independent samples $t$-test). Statistical analysis was performed using SPSS software version 20 and a statistically significant level of 0.05 was considered.

\section{Ethical Considerations}

After receiving a written letter of introduction and written consent from the selected researcher centers, initial measures were taken to carry out the project. All patients' information was kept confidential. All ethics statements were included in the study by regarding the declarations of Helsinki and Ethics Research Committees of the University of Medical Sciences. The project was carried out after approval by the Research Council of the Medical School and receiving the code of ethic (IR.ZAUMS.REC.1398.199 and IRCT20190813044521N1).

\section{Results}

In this study, 90 patients were included in the study. The mean age in the case group was $28.11 \pm 7.24$ years and in the control group was $29.16 \pm 8.45$ years. Statistically, the two groups did not differ significantly $(P=0.53)$. Also, no statistically significant difference was found between the two groups in terms of gender frequency $(P=0.82)$, (Table 1$)$.

The mean propofol consumption was $2.86 \pm 0.25 \mathrm{mg} / \mathrm{kg}$ in the case group and $3.01 \pm 0.4 \mathrm{mg} / \mathrm{kg}$ in the control group. The amount of propofol consumed in the case group was significantly lower than the control group $(P=0.039)$. The amount of propofol consumed by sex in the study groups was also calculated. As shown in Table 2, the amount of propofol consumed in men and women was not statistically significant $(P<0.05)$.

Table 3 and Figure 1 compare the amount of MAP changes at the time before and after induction, immediately and 5 minutes after intubation in the two groups. Mean arterial blood pressure before induction was not found to be statistically significant between the two groups $(P=0.83)$. Statistical analysis of data by ANOVA test did not show a significant interaction between time and group $(P=0.217$ and $P=0.86)$, but a statistically significant difference was found in mean arterial blood pressure of patients during the 4 time points $(F=7.59$ and $P=0.001)$. Furthermore, a significant increase was found in

\begin{tabular}{ccccc}
\hline \multicolumn{4}{c}{ Table 1. Gender frequency of patients } \\
\cline { 3 - 4 } & \multicolumn{3}{c}{ Sex } & \multirow{2}{*}{ Total } \\
\cline { 2 - 4 } & Male & Female & \\
\hline \multirow{2}{*}{ Group } & Case & $28(62.2 \%)$ & $17(37.8 \%)$ & $45(100 \%)$ \\
& Control & $29(64.4 \%)$ & $16(35.6 \%)$ & $45(100 \%)$ \\
& & 0.82 & \\
\hline
\end{tabular}




\begin{tabular}{|c|c|c|c|}
\hline Group & Sex & Propofol dose (mg/kg) & $P$-value ${ }^{*}$ \\
\hline \multirow{2}{*}{ Case } & Male & $2.86 \pm 0.29$ & \multirow{2}{*}{0.94} \\
\hline & Female & $2.85 \pm 0.19$ & \\
\hline \multirow{2}{*}{ Control } & Male & $3.02 \pm 0.45$ & \multirow{2}{*}{0.73} \\
\hline & Female & $2.98 \pm 0.3$ & \\
\hline \multirow{2}{*}{ Total } & Male & $2.94 \pm 0.38$ & \multirow{2}{*}{0.68} \\
\hline & Female & $2.91 \pm 0.25$ & \\
\hline
\end{tabular}

*Independent samples $t$-test.

\begin{tabular}{|c|c|c|c|}
\hline \multirow{2}{*}{ Evaluation time } & \multicolumn{2}{|c|}{ Group } & \multirow{2}{*}{$P$-value ${ }^{*}$} \\
\hline & Case & Control & \\
\hline Before induction & $9.78 \pm 1.3$ & $9.27 \pm 1.3$ & \\
\hline After induction & $9.28 \pm 1.03$ & $9.09 \pm 1.11$ & \\
\hline $\begin{array}{l}\text { Immediately after } \\
\text { intubation }\end{array}$ & $9.03 \pm 1.34$ & $8.74 \pm 1.28$ & 0.86 \\
\hline $\begin{array}{l}5 \text { minutes after } \\
\text { intubation }\end{array}$ & $9.94 \pm 1.43^{* * *}$ & $9.54 \pm 1.3^{* *}$ & \\
\hline
\end{tabular}

*Mixed ANOVA test. ${ }^{*} P<0.05$.

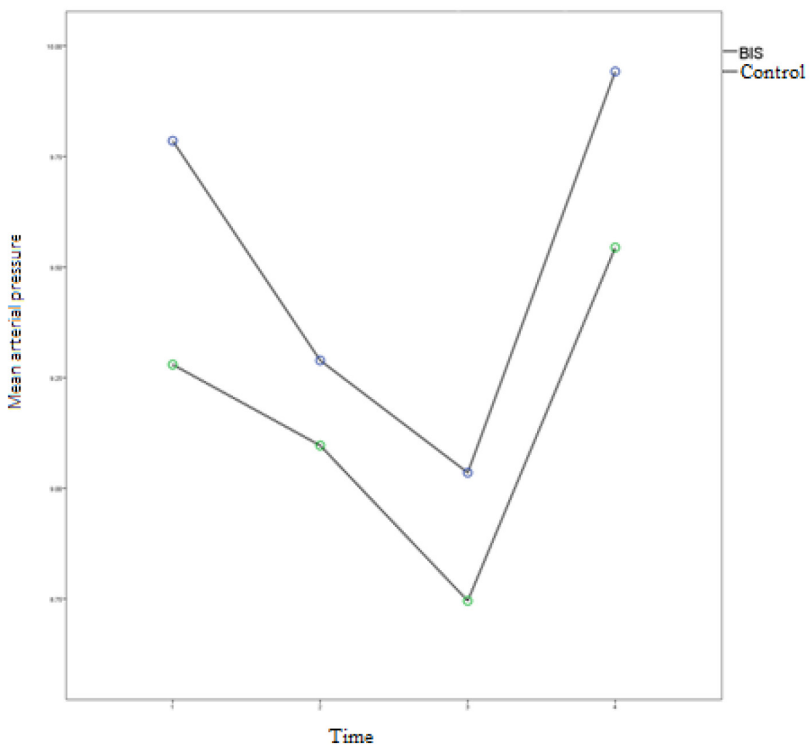

Fig. 1 Changes in mean arterial pressure at different times.

mean arterial pressure after intubation in both groups (case group: $P=0.011$; control group: $P=0.034$ ).

The comparisons of heart rate changes at times before and after induction, immediately and 5 minutes after intubation in the two groups are shown in Table 4 and Figure 2. There was no statistically significant difference in heart rate between patients before induction $(P=0.48)$. Statistical analysis of data by ANOVA test did not show a significant interaction between time and group $(P=0.418$ and $P=0.74)$. However, a statistically significant difference was found in patients' heart rate during the 4 time points $(F=7.59$ and $P=0.001)$. A significant increase was found in heart rate after intubation in both groups $(P=0.001)$.

\begin{tabular}{llll}
\hline \multicolumn{3}{l}{ Table 4. Heart rate at different times } \\
\hline \multirow{2}{*}{ Evaluation time } & \multicolumn{2}{c}{ Group } & \multirow{2}{*}{ P-value* $^{*}$} \\
\cline { 2 - 3 } & Case & Control & \\
\hline Before induction & $80.13 \pm 4.87$ & $80.17 \pm 5.39$ & \\
After induction & $81.93 \pm 4.22$ & $81.46 \pm 4.04$ & \\
Immediately after & $80.75 \pm 6.64$ & $79.84 \pm 4.67$ & 0.74 \\
intubation & & & \\
5 minutes after & $86.77 \pm 4.14^{* * *}$ & $87.35 \pm 4.14^{* *}$ & \\
intubation & & & \\
\hline
\end{tabular}

*Mixed ANOVA test.

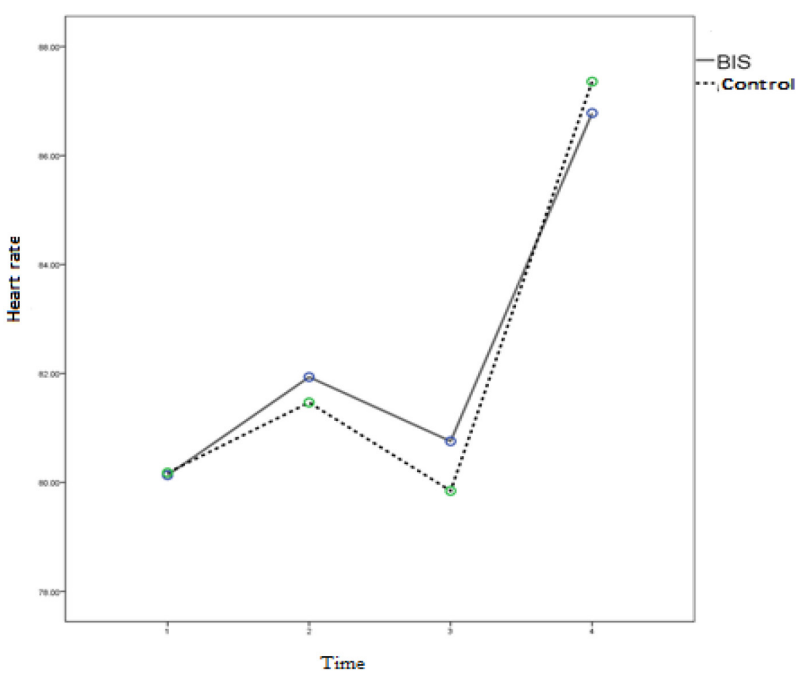

Fig. 2 Heart rate changes at different times.

\section{Discussion}

Propofol is a new intravenous hypnotic drug that is widely used in anesthesia. Monitoring the depth of anesthesia with BIS is also a parameter derived from electroencephalography (EEG), which is associated with sleep deprivation and loss of consciousness. ${ }^{15}$

Therefore, the aim of this study was to evaluate the effect of using BIS monitoring during induction of anesthesia on the amount of propofol consumed in patient candidates for surgery. In the present study, no significant difference was observed between the mean age and gender $(P>0.05)$. The amount of propofol consumed in the case group was significantly lower than the control group $(P=0.039)$ and the amount of propofol consumed in men and women was not found to be statistically significant $(P<0.05)$. Mean arterial blood pressure before induction was not statistically significant between the two groups $(P=0.83)$. Statistical analysis of data by ANOVA test did not show a significant interaction between time and group $(P=0.217$ and $P=0.86)$, but a statistically significant difference was observed in mean arterial blood pressure of patients during the 4 time points $(P=0.0011)$. In addition, a significant increase was found in mean arterial pressure after intubation in both groups $(P=0.011$ and $P=0.034)$.

There was no statistically significant difference in heart rate between patients before induction $(P=0.48)$. Statistical analysis of data by ANOVA test did not reveal a significant correlation between time and group $(P=0.418$ and $P=0.74)$. But a statistically significant difference was observed in 
patients' heart rate during the 4 time points $(F=7.59$ and $P=$ $0.001)$. There was a significant increase in heart rate after intubation in both groups $(P=0.001)$.

In this regard, various studies have been conducted. For instance, Mahori et al., evaluated the effect of BIS index on propofol consumption in patients undergoing coronary artery bypass graft surgery and stated that although propofol consumption in the intervention group was less than control group but this finding was not statistically significant and the time of endotracheal tube removal after surgery was not found to be statistically significant between the two groups. This study stated that the binomial index is a valuable tool for monitoring the depth of anesthesia, but can not be useful as a guide for propofol administration. ${ }^{17}$ Arbabpour et al. Showed that the BIS index for as monitoring the depth of anesthesia in cesarean section leads to acceleration of the time of removal of the endotracheal tube, and time of recovery, as well as acceleration of the mother's consciousness to care for the baby. ${ }^{18}$ The results of the evaluation are consistent with the present study.

Hasani et al. also evaluated the effect of constant dose and variable dose of propofol maintenance on waking time by monitoring the BIS index. According to the results, the time of the first awakening response in the first group who received the variable dose of propofol was 480 seconds on average while it was found to be 549 seconds in the second group was $(P<0.05)$. The total amount of drug used during surgery, which lasted about $120 \pm 30$ minutes for all patients, averaged $980 \mathrm{mg}$ in the first group and $1240 \mathrm{mg}$ in the second group. Accordingly, administration of propofol infusion by monitoring the depth of anesthesia with BIS index during anesthesia accelerates recovery and reduces propofol consumption. ${ }^{19}$ This is also consistent with the results of the present study.

Another study by Hasani et al. reported no statistically significant difference in the effect of vital signs in the study population and the time to general anesthesia with sevoflurane was less than that of isoflurane and halothane, where sevoflurane was more suitable for general anesthesia in children. ${ }^{20}$ Kamali et al. also examined the effect of BIS on patients undergoing cesarean section and showed that the level of awareness during anesthesia was significantly higher in the control group than the BIS group $(P=0.0001)$. Accordingly, the incidence of awareness during anesthesia in the group with BIS monitor is significantly lower than the control group. ${ }^{21}$ This finding was also consistent with and confirming our findings.

Other findings of the present study showed a significant increase in mean arterial blood pressure and heart rate in both groups after intubation. Ko et al. reported a similar finding where an increase in blood pressure and heart rate was found after intubation. ${ }^{22}$ Tabari et al. compared hemodynamic changes during anesthesia between tracheal intubation and laryngeal mask use. They also reported similar results on moderate arterial hypertension in both groups, but no statistically significant difference was found between the two groups in this regard. ${ }^{23}$ In general, various studies, including the present study, have shown that the use of BIS can be effective in improving the condition of patients, however, there is a need for more studies in this area.

\section{Conclusion}

The use of BIS can be effective in reducing the amount of propofol intake and its side effects. The condition of patients under general anesthesia can be improved by BIS, resulting in improvement of the next condition.

\section{Conflicts of Interest}

None.

\section{References}

1. Adrian W. Gelb, Kate Leslie, Donald R. Stanski, Steven L. Shafers. Monitoring the depth of Anesthesia. In: Miller's Anesthesia $7^{\text {th }}$ ed, churchill livingstone, USA, 2010. p:1245

2. Fahy BG, Chau DF. The Technology of Processed Electroencephalogram Monitoring Devices for Assessment of Depth of Anesthesia. Anesthesia and analgesia. 2018;126(1):111-7.

3. Ferreira A, Nunes C, Ferreira AL, Tedim R, Amorim P. Inter-patient variability and predictive factors of propofol requirements and estimated concentrations for loss of consciousness and recovery. J Neurosurg Anesthesiol. 2015;27:260-1.

4. Ferreira AL, Mendes JG, Nunes CS, Amorim P. Evaluation of Bispectral Index time delay in response to anesthesia induction: an observational study. Brazilian J Anesthesiol Elsevier. 2019;69:377-82

5. Upton RN, Martinez AM, Grant C. Comparison of the sedative properties of CNS 7056, midazolam, and propofol in sheep. British journal of anaesthesia. 2009;103(6):848-57

6. Bijker JB, Persoon S, Peelen LM, Moons KG, Kalkman CJ, Kappelle LJ, et al. Intraoperative hypotension and perioperative ischemic stroke after general surgery: a nested case-control study. Anesthesiology. 2012;116(3):658-64.

7. Walsh M, Devereaux PJ, Garg AX, Kurz A, Turan A, Rodseth RN, et al. Relationship between intraoperative mean arterial pressure and clinical outcomes after noncardiac surgery: toward an empirical definition of hypotension. Anesthesiology. 2013;119(3):507-15.

8. MonkTG, Saini V, Weldon BC, Sigl JC. Anesthetic management and one-year mortality after noncardiac surgery. Anesthesia and analgesia. 2005;100(1):4-10.

9. Sepúlveda PO, Carrasco E, Tapia LF, Ramos M, Cruz F, Conget $\mathrm{P}$, et al. Evidence of hysteresis in propofol pharmacodynamics. Anaesthesia. 2018;73:40-8.
10. Gonzalez-Cava JM, Reboso JA, Calvo-Rolle JL, Mendez-Perez JA. Adaptive drug interaction model to predict depth of anesthesia in the operating room. Biomed Signal Process Control. 2020;59:101931.

11. Vakkuri A, Yli-Hankala A, Talja P, Mustola S, Tolvanen-Laakso H, Sampson T, et al. Time-frequency balanced spectral entropy as a measure of anesthetic drug efect in central nervous system during sevofurane, propofol, and thiopental anesthesia. Acta Anaesthesiol Scand. 2004:48:145-53.

12. Mourisse J, Lerou J, Struys M, Zwarts M, Booij L. Multi-level approach to anaesthetic efects produced by sevofurane or propofol in humans: 1. BIS and blink refex. Br J Anaesth. 2007;98:737-45.

13. Scott Al. Electroconvulsive therapy, practice and evidence. Br J Psychiatry. 2010;196(3):171-172.

14. Kimball JN, Rosenquist PB, Dunn A, McCall V. Prediction of antidepressant response in both 2.25xthreshold RUL and fixed high dose RUL ECT. J Affect Disord. 2009;112(1-3):85-91.

15. Castro A, Bressan N, Lobo F, Nunes C, Amorim P. The higher the propofol concentration needed for loss of consciousness the larger its diference to the concentrations required at maintenance, using TCI and BIS guided anesthesia. Eur J Anaesthesiol. 2008;25:150.

16. Arya S, Asthana V, Sharma JP. Clinical vs. bispectral index-guided propofol induction of anesthesia: A comparative study. Saudi journal of anaesthesia. 2013;7(1):75-9

17. Mahori A, Heshmati F, Abbasivash R, Norozinia H, Hassani E, Nasiri AA. The effect of Bispectral Index monitoring on propofol consumption in patients undergoing coronary artery bypass graft. Studies in Medical Sciences. 2011;22(3):249-54.

18. Arbabpour R, Ganji Fard M, Tabiee S, Saadatjoo SA. The effect of Bi-Spectral Index on recovery and postoperative complications in patients undergoing caesarean section. Journal of Birjand University of Medical Sciences. 2015:22(2):94-103. 
19. Hasani V, Zamani F, Khosravi A. Comparison of constant dose with variable dose of propofol for studying time of wake up within Bispectral index (BIS) monitoring. Razi Journal of Medical Sciences. 2003;9(31):509-14.

20. Hassani V, Javanbakht $\mathrm{S}$. Comparison of effects of Halothane, Isoflurane and Sevoflurane on depth of anesthesia using BIS monitoring in cochlear implantation of children aged 2-6 years old, in Hazrat-e Rasool Akram hospital. Razi Journal of Medical Sciences. 2007;14(55): 89-94.

21. Kamali A, Shokrpour M, Pazoki S, Moshiri E, Taheri-Nejad M, Dadashpour N, et al. Determining the effect of BIS monitoring on level of awareness during anesthesia in women undergoing elective caesarean section. Journal of Arak University of Medical Sciences. 2015;17(12):56-63.

22. Ko DD, Kang H, Yang SY, Shin HY, Baek CW, Jung YH, et al. A comparison of hemodynamic changes after endotracheal intubation by the Optiscope and the conventional laryngoscope. Korean journal of anesthesiology. 2012;63(2):130-5

23. Tabari M, Alipour M, Ahmadi M. Hemodynamic changes occurring with tracheal intubation by direct laryngoscopy compared with intubating laryngeal mask airway in adults: A randomized comparison trial. Egyptian Journal of Anaesthesia. 2013;29(2):103-7.

This work is licensed under a Creative Commons Attribution-NonCommercial 3.0 Unported License which allows users to read, copy, distribute and make derivative works for non-commercial purposes from the material, as long as the author of the original work is cited properly. 